\title{
Electrostatic Aggregation and Formation of Core-Shell Suprastructures in Binary Mixtures of Charged Metal Nanoparticles
}

\author{
Alexander M. Kalsin ${ }^{1}$, Maciej Paszewski ${ }^{1}$, Anatoliy O. Pinchuk ${ }^{2}$, George C. Schatz ${ }^{2}$ and Bartosz A. \\ Grzybowski ${ }^{1 *}$ \\ ${ }^{1}$ Department of Chemical and Biological Engineering and The Northwestern Institute on Complex \\ Systems; Northwestern University, 2145 Sheridan Rd., Evanston, IL 60208
}

${ }^{2}$ Department of Chemistry; Northwestern University, 2145 Sheridan Rd., Evanston, IL 60208

* Corresponding author - Email: grzybor@northwestern.edu; Tel. 847.491.3024

1. Synthesis of Nanoparticles. Dodecylamine (DDA) coated gold and decanoic acid (DA) coated silver nanoparticles were prepared according to a modified literature procedure [Jana, N. R.; Peng, X. G. J. Am. Chem. Soc. 2003, 125, 14280]. For AuNPs, we used $\mathrm{HAuCl}_{4} \cdot 3 \mathrm{H}_{2} \mathrm{O}$ instead of $\mathrm{AuCl}_{3}$ to obtain smaller AuDDA particles (average size $\langle d\rangle=5.1 \mathrm{~nm}$; dispersity $\sigma=20 \%$ ). AgDA NPs in toluene were kept overnight to precipitate larger particles; the supernatant was then filtered through a $0.22 \mu \mathrm{m}$ syringe driven filter to give $\langle d\rangle=4.8 \mathrm{~nm}$ and $\sigma=30 \%$ particles. The average sizes of the particles were estimated based on high-resolution TEM images of at least 500 NPs of each type. Gold and silver NPs were functionalized with 11-mercaptoundecanoic acid (MUA) and N,N,N-trimethyl(11mercaptoundecyl)ammonium chloride (TMA). The ultrapure-grade MUA and TMA thiols were 
obtained from ProChimia (www.prochimia.com) and used as received.

1a. Ligand exchange on gold NPs. A toluene solution of DDA capped gold particles ( $7 \mu \mathrm{mol} / \mathrm{ml}, 20$ $\mathrm{ml}, 140 \mu \mathrm{mol})$ was quenched with $100 \mathrm{ml}$ of methanol to give a black precipitate, the supernatant solution with excess of capping agent and surfactant was decanted. The precipitate was washed with methanol $(3 \times 30 \mathrm{ml})$, dissolved in toluene $(50 \mathrm{ml})$, to which a methanolic solution of the thiol ligand was added upon stirring. The solutions of thiols were prepared as follows: $40 \mathrm{mg}(140 \mu \mathrm{mol})$ of TMA was dissolved in $10 \mathrm{ml}$ of methanol or $30 \mathrm{mg}(140 \mu \mathrm{mol})$ of MUA was initially neutralized with tetramethylammonium hydroxide (25\% methanolic solution, $70 \mu \mathrm{L}, 165 \mu \mathrm{mol})$ and then dissolved in 10 $\mathrm{ml}$ of methanol. The precipitate of thiol coated gold NPs was allowed to settle down, the mother liqueur solution was decanted, and the solid was washed with methanol (MUA coated NPs) or with ethyl acetate (TMA coated NPs). Then the precipitate was dissolved in $5 \mathrm{ml}$ of either methanol (AuTMA) or deionized water (AuMUA) and precipitated with $100 \mathrm{ml}$ of ethyl acetate (AuTMA) or acetone (AuMUA). The supernatant was discarded and the precipitate was washed with ethyl acetate and methanol, respectively. Finally, all the precipitates of thiol coated gold NPs were dried and dissolved in $14 \mathrm{ml}$ of water to obtain a $10 \mathrm{mM}$ solution of NPs. In the case of AuMUA the $\mathrm{pH}$ of the solution was adjusted to $\sim 11$ with $25 \%$ methanolic solution of $\mathrm{NMe}_{4} \mathrm{OH}$.

1b. Ligand exchange on silver NPs. To a toluene solution of DA coated silver NPs $(\sim 6 \mu \mathrm{mol} / \mathrm{ml}, 50$ $\mathrm{ml}, \sim 300 \mu \mathrm{mol})$ was added a solution of thiol ligand in $10 \mathrm{ml}$ of methanol: either TMA (84 mg, $300 \mu \mathrm{mol})$ or MUA $(65 \mathrm{mg}, 300 \mu \mathrm{mol})$ neutralized with $25 \%$ methanolic solution of $\mathrm{NMe}_{4} \mathrm{OH}(150 \mu \mathrm{L}$, $350 \mu \mathrm{mol})$. The dark brown precipitate was separated, then washed $(5 \times 30 \mathrm{ml})$ with either ethyl acetate or methanol for AgTMA and AgMUA, respectively. The AgTMA precipitate was redispersed in $5 \mathrm{ml}$ of methanol and quenched again with ethyl acetate, then washed with ethyl acetate and dried. Similarly, the AgMUA precipitate was redispersed in $5 \mathrm{ml}$ of water followed by precipitation with acetone (100 $\mathrm{ml})$, and the precipitate was then washed with methanol $(5 \times 30 \mathrm{ml})$ and dried. Yields: AgTMA $(33 \mathrm{mg}$, 
$264 \mathrm{mmol}$, content of thiol is about $20 \%$, calculated according to the formula [Stoeva, S., Klabunde, K. J., Sorensen, C. M. \& Dragieva, I. Gram-scale synthesis of monodisperse gold colloids by the solvated metal atom dispersion method and digestive ripening and their organization into two- and threedimensional structures. J. Am. Chem. Soc. 124, 2305-2311 (2002).]; AgMUA (27 mg, 216 mmol, content of thiol is about $20 \%$, calculated according to the same formula. The dried AgTMA and AgMUA were then dissolved in $13.2 \mathrm{ml}$ and $10.6 \mathrm{ml}$ of deionized water, respectively to obtain $20 \mathrm{mM}$ solutions. The $\mathrm{pH}$ of the AgMUA solution was adjusted to $\sim 11$ by adding $25 \%$ methanolic solution of $\mathrm{NMe}_{4} \mathrm{OH}$.

All thus prepared solutions of thiol coated gold and silver NPs were used as stock solutions for preparing $2 \mathrm{mM}$ solutions for titrations.

2. Estimation of NP Compositions. For silver NPs the surface area occupied by a single thiol molecule on silver nanoparticles was assumed to be the same as for gold nanoparticles, since both gold and silver NPs have $f C c$ crystal packing and their lattice constants are almost identical, namely $4.07 \AA$ and $4.08 \AA$, respectively)

3. UV-Vis titration. The UV-Vis spectra were recorded on a Cary Model 1 UV-VIS spectrophotometer in the range $300-800 \mathrm{~nm}$ in an optical glass cell (1mm of path length). The initial concentrations of both AuMUA $\left(c_{A u}^{0}\right)$ and AgTMA $\left(c_{A g}^{0}\right)$ aqueous solutions were $2 \mathrm{mM}$. Solutions of AuNPs $(400 \mu \mathrm{L}, 0.8 \mu \mathrm{mol})$ were titrated in a stirred vial by adding $0.05-0.1$ equivalent aliquots $(20-40$ $\mu \mathrm{L}$ ) of AgNPs solution, or for the reverse process, AuNPs were added to AgNPs. The titrations were carried out in a stirred vial, and after each addition the solution was allowed to equilibrate for 5 min, and then transferred to a UV-VIS cell. The molar absorption coefficients of gold, $\varepsilon_{A u}\left(\lambda_{\max , A u}\right)$, and silver, $\varepsilon_{A g}\left(\lambda_{\text {max }, A g}\right)$, were defined for each titration point using a procedure described below.

4. Derivation and Explanation of Absorption Coefficients. The absorption coefficients of gold 
$\varepsilon_{A u}\left(\lambda_{\max , A u}\right)$ and silver $\varepsilon_{A g}\left(\lambda_{\text {max }, A g}\right)$ were defined at the maxima of the gold $\lambda_{\text {max }, A u}$ and silver $\lambda_{\max , A g}$ plasmon bands, respectively, and were derived from the Lambert-Beer law in which the absorbance of a solution $A b s=\varepsilon c l$ is proportional to the molar absorption coefficient $\left(\varepsilon, \mathrm{M}^{-1} \cdot \mathrm{cm}^{-1}\right)$, concentration $(c$, $\mathrm{M})$, and the path length of the cuvette $(l, \mathrm{~cm})$.

If two solutions of non-interacting NPs are mixed, the total absorbance of the resulting mixture should be given by the sum of absorbances of gold and silver NPs in this mixture: $A b s=A b s_{A u}+A b s_{A g}=\varepsilon_{A u} \cdot c_{A u} \cdot l+\varepsilon_{A g} \cdot c_{A g} \cdot l$, where $c_{A u}$ and $c_{A g}$ are the concentrations of, respectively, AuNPs and AgNPs in a mixed solution. These concentrations can be expressed in terms of the mole fraction of AgNPs $\chi_{A g}$ present in the mixture as follows: $c_{A u}=c_{A u}^{0} /\left(1+\frac{\chi_{A g}}{1-\chi_{A g}} \cdot \frac{c_{A u}^{0}}{c_{A g}^{0}}\right)$ and $c_{A g}=c_{A u}^{0} /\left(\frac{\chi_{A g}}{1-\chi_{A g}}+\frac{c_{A u}^{0}}{c_{A g}^{0}}\right)$, where $c_{A u}^{0}$ and $c_{A g}^{0}$ are the initial concentrations of, respectively, gold and silver NPs in isolated (i.e., before mixing) solutions. For the equal initial concentrations used in our experiments, $c_{A u}^{0}=c_{A g}^{0}=c^{0}$, the expressions simplify to $c_{A u}=\left(1-\chi_{A g}\right) \cdot c^{0}$ and $c_{A g}=\chi_{A g} \cdot c^{0}$. This gives the total absorbance the wavelength $\lambda_{\max , A u}$ : $\operatorname{Abs}\left(\lambda_{\max , A u}\right)=\varepsilon_{A u}\left(\lambda_{\max , A u}\right) \cdot c^{0} \cdot\left(1-\chi_{A g}\right) \cdot l+\varepsilon_{A g}\left(\lambda_{\max , A u}\right) \cdot c^{0} \cdot \chi_{A g} \cdot l, \quad$ where $\varepsilon_{A g}\left(\lambda_{\max , A u}\right) \cdot c^{0} \cdot l=A b s_{A g}^{0}\left(\lambda_{\max , A u}\right)-$ the absorbance of a solution of AgNPs of the initial concentration $c^{0}$ at $\lambda_{\max , A u}$. Hence, the absorption coefficient of gold NPs defined at $\lambda_{\max , A u}$ is $\varepsilon_{A u}\left(\lambda_{\max , A u}\right)=\frac{1}{\left(1-\chi_{A g}\right) \cdot c^{0} \cdot l} \cdot\left[A b s\left(\lambda_{\max , A u}\right)-A b s_{A g}^{0}\left(\lambda_{\max , A u}\right) \cdot \chi_{A g}\right]$. The absorption coefficient of silver was derived analogously to give $\varepsilon_{A g}\left(\lambda_{\max , A g}\right)=\frac{1}{l \cdot c^{0} \cdot \chi_{A g}} \cdot\left[\left(A b s\left(\lambda_{\max , A g}\right)-A b s_{A u}^{0}\left(\lambda_{\max , A g}\right) \cdot\left(1-\chi_{A g}\right)\right]\right.$, where $A b s_{A u}^{0}\left(\lambda_{\max , A g}\right)$ is the 
absorbance of a solution of AuNPs of concentration $c^{0}$, and $A b s\left(\lambda_{\max , A g}\right)$ is the total absorbance of a mixture of AuNPs and AgNPs at wavelength $\lambda_{\max , A g}$.

Similarly, for "reverse" titrations - that is, when AuNPs were added to AgNPs -- the concentrations change in the following way $c_{A u}=\chi_{A g} \cdot c^{0}$ and $c_{A g}=\left(1-\chi_{A g}\right) \cdot c^{0}$, and the equations for the absorption coefficients $\quad$ are $\quad \varepsilon_{A u}\left(\lambda_{\max , A u}\right)=\frac{1}{l \cdot c^{0} \cdot\left(1-\chi_{A g}\right)} \cdot\left[\left(A b s\left(\lambda_{\max , A u}\right)-A b s_{A g}^{0}\left(\lambda_{\max , A u}\right) \cdot \chi_{A g}\right] \quad\right.$ and $\varepsilon_{A u}\left(\lambda_{\max , A u}\right)=\frac{1}{\chi_{A g} \cdot c^{0} \cdot l} \cdot\left[A b s\left(\lambda_{\max , A g}\right)-A b s_{A u}^{0}\left(\lambda_{\max , A g}\right) \cdot\left(1-\chi_{A g}\right)\right]$.

We make two important remarks:

First, we emphasize that the expressions for $\varepsilon_{A u}\left(\lambda_{\max , A u}\right)$ and $\varepsilon_{A g}\left(\lambda_{\max , A g}\right)$ remain finite and are thus valid only for, respectively, $\chi_{A g}<1$ and $\chi_{A g}>0$. Second, we note that our construction assumes that the absorption coefficients of silver $\varepsilon_{A g}\left(\lambda_{\max , A u}\right)=\frac{A b s_{A g}^{0}\left(\lambda_{\max , A u}\right)}{l_{A g} \cdot c^{0}}$ at $\lambda_{\max , A u}$ and of gold $\varepsilon_{A u}\left(\lambda_{\max , A g}\right)=\frac{A b s_{A u}^{0}\left(\lambda_{\text {max }, A g}\right)}{l \cdot c^{0}}$ at $\lambda_{\text {max }, A g}$ are constant during the titration. 